\title{
The Gaia mission: science, organization and present status
}

\author{
L. Lindegren ${ }^{1}$, C. Babusiaux ${ }^{2}$, C. Bailer-Jones ${ }^{3}$ U. Bastian ${ }^{4}$, A. G. A. \\ Brown ${ }^{5}$, M. Cropper ${ }^{6}$, E. Høg ${ }^{7}$, C. Jordi ${ }^{8}$, D. Katz ${ }^{2}$, F. van Leeuwen ${ }^{9}$, \\ X. Luri ${ }^{8}$, F. Mignard ${ }^{10}$, J. H. J. de Bruijne ${ }^{11}$ and T. Prusti ${ }^{11}$ \\ ${ }^{1}$ Lund Observatory, Lund University, Sweden, email: lennart@astro.lu.se \\ ${ }^{2}$ Observatoire de Paris, Meudon, France \\ email: carine.babusiaux@obspm.fr, david.katz@obspm.fr \\ ${ }^{3}$ Max-Planck-Institut für Astronomie, Heidelberg, Germany, email: calj@mpia-hd.mpg.de \\ ${ }^{4}$ Astronomische Rechen-Institut, Heidelberg, Germany, email: bastian@ari.uni-heidelberg.de \\ ${ }^{5}$ Leiden Observatory, Leiden University, The Netherlands, email: brown@strw.leidenuniv.nl \\ ${ }^{6}$ Mullard Space Science Laboratory, Dorking, United Kingdom, email: msc@mssl.ucl.ac.uk \\ ${ }^{7}$ Niels Bohr Institute, Copenhagen, Denmark, email: erik@astro.ku.dk \\ ${ }^{8}$ University of Barcelona - IEEC, Barcelona, Spain, email: carme@am.ub.es, xluri@am.ub.es \\ ${ }^{9}$ Institute of Astronomy, Cambridge, United Kingdom, email: fvl@ast.cam.ac.uk \\ ${ }^{10}$ Observatoire de la Côte d'Azur, Nice, France, email: francois.mignard@obs-nice.fr \\ ${ }^{11}$ ESA, ESTEC, Noordwijk, The Netherlands \\ email: Jos.de.Bruijne@rssd.esa.int, Timo.Prusti@rssd.esa.int
}

\begin{abstract}
The ESA space astrometry mission Gaia will measure the positions, parallaxes and proper motions of the 1 billion brightest stars on the sky. Expected accuracies are in the $7-25 \mu$ as range down to $15 \mathrm{mag}$ and sub-mas accuracies at the faint limit $(20 \mathrm{mag})$. The astrometric data are complemented by low-resolution spectrophotometric data in the 330-1000 nm wavelength range and, for the brighter stars, radial velocity measurements. The scientific case covers an extremely wide range of topics in galactic and stellar astrophysics, solar system and exoplanet science, as well as the establishment of a very accurate, dense and faint optical reference frame. With a planned launch around 2012 and an (extended) operational lifetime of 6 years, final results are expected around 2021. We give a brief overview of the science goals of Gaia, the overall project organisation, expected performance, and some key technical features and challenges.
\end{abstract}

Keywords. astrometry, Galaxy: kinematics and dynamics, planetary systems, relativity, space vehicles, stars: fundamental parameters, techniques: photometric, techniques: radial velocities

\section{Scientific case}

The scientific case for Gaia rests on the extremely powerful combination of three distinct qualities in a single mission: (i) the ability to make very accurate (global and absolute) astrometric measurements; (ii) the capability to survey large and complete (flux limited) samples of objects; (iii) the matching collection of synoptic, multi-epoch spectrophotometric and radial-velocity measurements. The range of topics that can be addressed with such a data set is too immense to be described here except in the most superficial and incomplete way. Below we highlight the expectations in four different areas. For more detailed reviews on a much broader variety of topics we refer to the proceedings of the symposium The Three-Dimensional Universe with Gaia (Turon et al. 2005).

Galactic astrophysics: Large, volume-complete samples allow the determination of spatially resolved statistics such as the luminosity and initial mass functions, star formation 
rates and stellar multiplicity. Distance information combined with stellar classification and photometry allows unprecedented three-dimensional mapping of interstellar extinction. Gaia is the first survey providing six-dimensional phase space $(\mathbf{r}, \mathbf{v})$ data coupled with photometry for very large, magnitude-limited samples. These are crucial for disentangling the complex relationships between the spatial and kinematic distributions of the stars and their ages and chemical enrichment, which encode the Galactic evolution. The number density and kinematics of tracer stars can be used to map the galactic potential over a large part of the Galaxy, thus determining the distribution of (dark) matter and shedding new light on disk dynamics (bar, spiral structure, warp). In the halo, phase space (or $E, L_{z}$ ) structures may reveal past galactic mergers (Aguilar et al. 2006).

Stellar astrophysics: Gaia will provide accurate distances to stellar clusters and individual stars, covering a very wide range of masses and evolutionary stages. Individual distances to better than $<1 \%$ will be obtained for some $10^{7}$ stars. Together with multi-wavelength spectrophotometry this provides accurate luminosity calibrations and stringent tests of stellar interior models, model atmospheres and stellar evolution. There is clearly an enormous scope for synergy with more specialised ground-based observations (high-resolution spectroscopy, astroseismology) and theoretical investigations. For intrinsically bright objects, a major part of the Galaxy can be reached with moderate distance errors (see Table 2) and fundamental parameters can be obtained for rare objects. For stars closer to the Sun, Gaia will provide a sensitive survey of (visible or invisible) companion objects, including thousands of exoplanets.

Solar system: Gaia will detect and measure asteroids down to 20 mag whenever they enter the fields of view, about 15 times per year, to $\sim 1$ mas accuracy per epoch. Accurate proper elements can be determined and dynamical families identified. The masses for about 100 asteroids can be determined from close encounters (Mouret et al. 2007). Other physical characteristics (size, spin, shape, taxonomic classification) will be obtained for thousands of objects. Observations at high ecliptic latitudes and to within $45^{\circ}$ from the Sun will probe populations in exotic orbits and inside the Earth's orbit. The observations of Kuiper Belt objects, where masses may be determined for detected binary objects, will provide a valuable contribution to the study of the outer solar system.

Reference frame and experimental relativity: The many accurate positions and proper motions define a dense (>1500 stars $\left.\mathrm{deg}^{-2}\right)$ net of reference objects. The stellar data are directly linked to the extragalactic reference frame through quasars (about 500,000

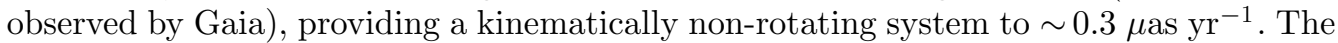
high accuracy of stellar proper motions means that the reference system is long-lived: e.g. 18 mag positions are good to $<1$ mas over the 40-year period 1995-2035. The quasar observations also give of the acceleration of the Solar System in a cosmological frame from the secular change in their stellar aberration (Bastian 1995). General relativity is consistently used for reducing the observations, but possible deviations can be tested through the gravitational light bending by the Sun and planets (sensitive to PPN $\gamma$ ) and the perihelion precession for about 300 asteroids (sensitive to PPN $\beta$ and solar $J_{2}$ ).

\section{Status and organization}

The Gaia satellite and mission operation is fully funded by the European Space Agency (ESA). The prime industrial contractor for building the satellite (including its scientific instruments), EADS Astrium, was selected in February 2006. The project passed the Preliminary Design Review in July 2007 and is now in the development and production Phase C/D with a launch target date in December 2011. Transfer to its orbit around the Sun-Earth $\mathrm{L}_{2}$ Lagrange point (1.5 million $\mathrm{km}$ from the Earth) and commissioning will 

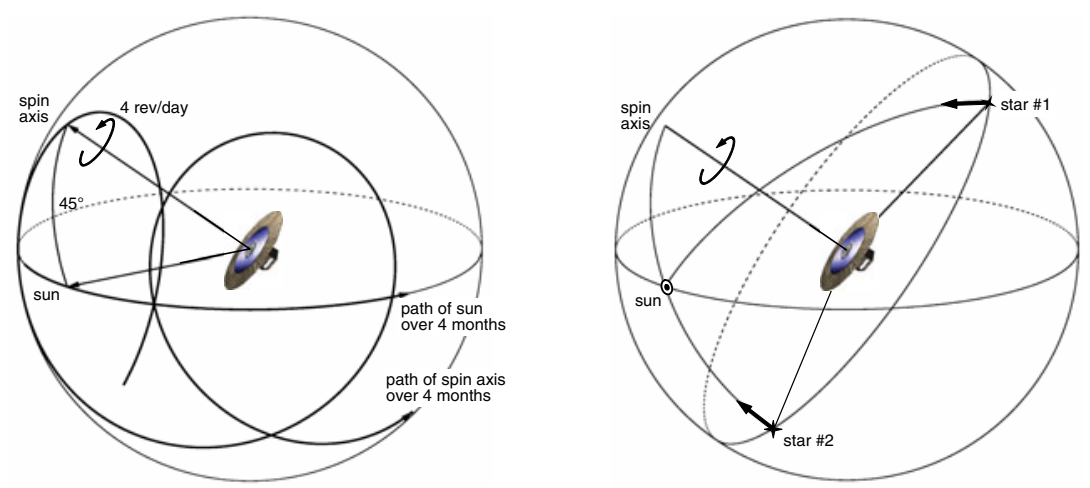

Figure 1. Left: Most of the sky is scanned in just a few months by the slowly spinning satellite, thanks to the precession of the spin axis about the satellite-sun direction. Right: The parallactic displacement of a star is directed along the great-circle arc from the star to the sun. The observed angle between the indicated stars (\#1 and \#2) consequently depends on the parallax of \#1, but is independent of the parallax of \#2. This makes it possible to derive absolute parallaxes.

take up to 6 months, after which the routine science operations phase starts. This phase will last 5 years, with a possible 1 year extension. The data processing will be on-going during the operation phase and for $2-3$ years after. The final catalogue is expected around 2021.0, but with intermediate data releases produced during the operational phase. The Data Processing and Analysis Consortium (DPAC) is described elsewhere (Mignard et al. 2008).

The overall scientific responsibility for the Gaia mission rests with the ESA Project Scientist, currently T. Prusti; until 2006 M.A.C. Perryman and during 2006 F. Jansen. The Project Scientist interfaces with a number of bodies both within and external to ESA, most importantly the Project Team at ESTEC (responsible for the procurement of the satellite, including its scientific instruments), the flight dynamics and mission operations teams at ESOC, the science operations team at ESAC, and DPAC. The Project Scientist chairs the Gaia Science Team (GST), a senior body in place since 2001 to provide ESA with advice on all aspects of the mission related to its scientific goals, performance and implementation. The present list of authors reflects the current composition of the GST (with J. de Bruijne a member of the Project Scientist's support team at ESTEC), but a very different team will soon become active, following an open invitation by ESA to submit proposals for membership in the GST.

The total human effort expended in the scientific preparations for Gaia is indicated by the list of persons currently involved in the Gaia project; currently (October 2007) this includes some 370 people, not counting the industrial contractors.

\section{Observation principle and main technical features}

Gaia builds on the proven principles of Hipparcos to determine the astrometric parameters (typically the position, parallax and proper motion of a star) for a large number of objects by combining a much larger number of essentially one-dimensional (along-scan) angular measurements in the focal plane. A continuous scanning motion (Fig. 1, left) similar to that of Hipparcos ensures that every object is observed in at least six distinct epochs per year in patterns that are geometrically favourable for the resolution of the astrometric parameters. The capability to make accurate differential measurements over long $\left(\sim 90^{\circ}\right)$ arcs is the key to obtaining absolute parallaxes (Fig. 1, right) as well as a globally consistent reference system for the positions and proper motions. Gaia has all of 

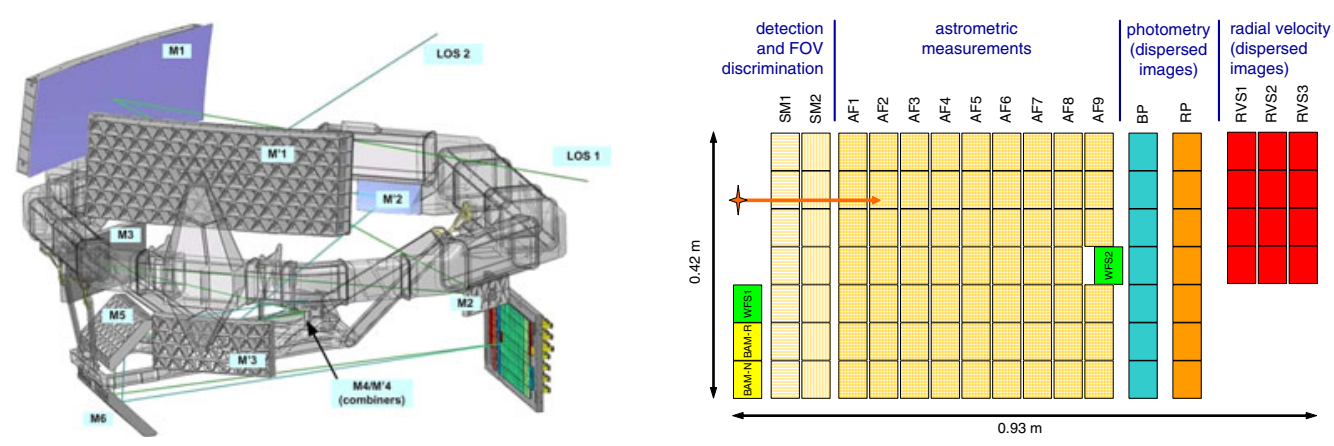

Figure 2. Left: The Gaia instrument, comprising two telescopes (mirrors M1-M6 and M'1-M6) with a common focal plane. M4/M'4 is the beam combiner situated at the exit pupil (EADS Astrium). Right: Layout of CCDs in the focal plane of Gaia. Images travel from left to right, crossing in turn the skymappers $\mathrm{SM}$, astrometric field $\mathrm{AF}$, blue photometer $\mathrm{BP}$, red photometer $\mathrm{RP}$, and (some of them) the radial-velocity spectrometer (RVS). Also shown are the CCDs for the basic-angle monitor (BAM) and wavefront sensors (WFS, for in-orbit optical adjustment).

these features in common with Hipparcos, although the details differ: the angle between the Sun and spin axis is $45^{\circ}$ (compared to $43^{\circ}$ for Hipparcos), the spin period is 6 hours (128 min for Hipparcos), and the basic angle is $106.5^{\circ}$ ( $58^{\circ}$ for Hipparcos).

The huge gain in accuracy, limiting magnitude and number of objects, compared with Hipparcos, is achieved by a combination of several factors: much larger optics (each of the two fields of view has an entrance pupil of $1.45 \times 0.5 \mathrm{~m}^{2}$; Fig. 2 , left) gives much improved resolution and photon collection; the CCDs have a wider spectral range and much better quantum efficiency than the photomultipliers of Hipparcos, as well as a huge multiplexing advantage, as tens of thousands of objects are observed in parallel. The CCDs have a pixel size of $10 \times 30 \mu \mathrm{m}^{2}$, matched to the optical resolution of the instrument and the effective focal length of $35 \mathrm{~m} . \dagger$

The autonomous on-board processing system detects any object brighter than $\simeq 20$ mag as it enters the focal plane, then tracks the object across a sequence of CCDs dedicated to the astrometric, photometric and radial-velocity measurements (Fig. 2, right). All the CCDs are operated in TDI mode. The astrometric CCDs sample a small window centred on the projected path of the optical image. In contrast to earlier designs of Gaia, the photometry is no longer made using filters, and the photometric and radial-velocity measurements share the same telescope and focal plane as the astrometry. These and other modifications (in early 2006) to the instrument have had a negative impact on the scientific performances, but were considered necessary in order to keep the project within given cost and mass constraints.

For the photometry there are thus two rows of CCDs, referred to as the blue and red photometers (BP and RP). Each of them is a slitless prism spectrophotometer, sampling the dispersed image in some 35 points covering the spectral interval $330-680 \mathrm{~nm}(\mathrm{BP})$ and $640-1050 \mathrm{~nm}(\mathrm{RP})$; see Fig. 3. The spectral resolution $R=\lambda / \Delta \lambda$ varies from 10 to 30 depending on the wavelength. Accumulated over the mission, any object will be observed $\sim 70$ times (average value) by each photometer.

Radial-velocity measurements will be made for stars brighter than $\sim 16.5$ mag using the slitless radial-velocity spectrometer (RVS). Its resolution $(R \simeq 11,500)$ and wavelength range $(847-874 \mathrm{~nm})$ have been optimised to allow radial velocities to be measured, at

$\dagger$ The enormous advantage of using CCDs for direct imaging in a scanning astrometric satellite was shown by Høg (1993) with the Roemer mission design. This efficient use of CCDs, later adopted for Gaia, was far from obvious to anyone at the time (Høg 2008). 

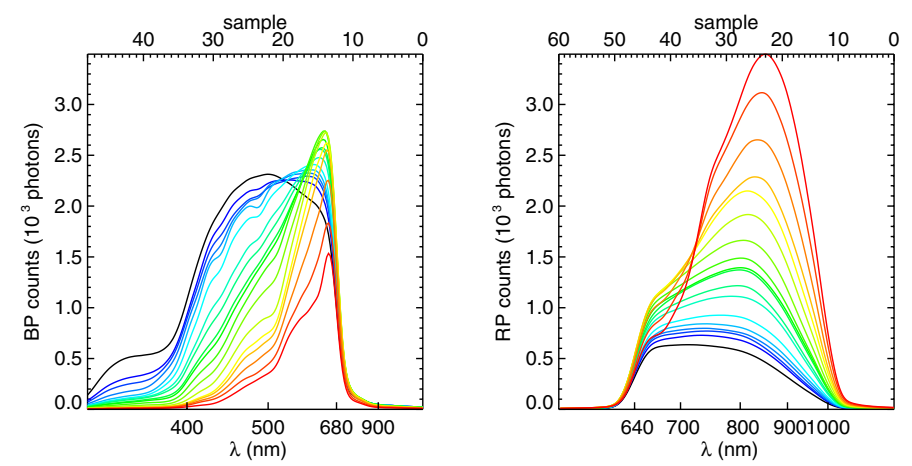

Figure 3. Simulated spectra of 18 main-sequence stars of spectral classes O8-M5, as observed in the blue and red photometers (BP, RP). The photon counts are normalised to the integrated Gaia magnitude $G=15$. Full PSF smearing effects are taken into account, but no noise has been added. (From Straizys et al. 2006.)

the $1-15 \mathrm{~km} \mathrm{~s}^{-1}$ accuracy level, for a wide range of spectral classes. It covers the Ca II triplet, which is strong for most intermediate to late type stars, as well as the hydrogen lines $\mathrm{P} 13-16$ dominating the early type spectra, and many weaker lines of various species. For a given object there will be on average $\sim 40$ transits across the three rows of CCDs used by the RVS.

\section{Calibration issues and predicted astrometric performance}

Table 1 summarizes the predicted astrometric performance for single, unperturbed solar-type stars. In Table 2 the predicted precision in parallax has been translated into distances for given relative error in parallax (or distance). Note that Gaia will observe virtually all stars of a given type and extinction, within their parallax horizon.

The predicted performance was calculated by means of the Gaia Accuracy Analysis Tool (de Bruijne 2005) using current (October 2007) mission and instrument data. The resulting rms errors include a science margin factor of 1.2. The accuracy analysis takes into account all identified relevant error sources, assuming that the engineering specifications of the individual components (CCD quantum efficiency, wavefront errors, etc) are met. While many of the specifications are technologically challenging, they are now all deemed realistic to achieve. However, the performance analysis also includes assumptions concerning the accuracy of critical instrument calibration parameters. These are normally derived as part of the scientific data processing itself, e.g. the precise geometrical layout of the CCDs. In a few cases the associated uncertainties are still considerable, because it has not yet been possible to formulate a complete calibration model.

By far the most difficult calibration issue at present concerns the radiation damage of the CCDs. It is well known that energetic particles in space (mainly protons from solar flares) may cause lattice defects in the silicon material where charges are transported during the CCD readout. As a result, some of the electrons generated by the optical image will be trapped by the defects, and randomly released at a later time. As a result, the electronic image may suffer a distortion, shift and charge loss compared to that of an undamaged CCD. In the RVS and photometers there will be a corresponding degradation in spectral fidelity and resolution. With the expected end-of-mission radiation dose, the systematic centroid shifts of the astrometric images may amount to several milliarcsec, depending in a complex way on many factors including the recent illumination history of the pixel column. The problem is aggravated by Gaia's launch near the next solar 
Table 1. Sky-averaged rms errors of the astrometric parameters for G0V stars (no extinction).

\begin{tabular}{lcrrrrrrr}
\hline$V$ magnitude & $6-13$ & 14 & 15 & 16 & 17 & 18 & 19 & 20 \\
\hline parallax [ $\mu$ as] & 8 & 13 & 21 & 34 & 55 & 90 & 155 & 275 \\
proper motion $\left[\mu\right.$ as yr $\left.{ }^{-1}\right]$ & 5 & 7 & 11 & 18 & 30 & 50 & 80 & 145 \\
position at mid-epoch $(\simeq 2015)[\mu$ as] & 6 & 10 & 16 & 25 & 40 & 70 & 115 & 205 \\
\hline
\end{tabular}

Table 2. Parallax horizon of Gaia, i.e. maximum distance (in $\mathrm{kpc}$ ) for given relative precision in distance (or parallax). Based on current accuracy estimates, with $M_{V}$ from Cox (2000).

\begin{tabular}{|c|c|c|c|c|c|c|c|c|c|c|}
\hline \multirow[b]{2}{*}{ Type of star } & \multicolumn{5}{|c|}{ no extinction $\left(A_{V}=0\right)$} & \multicolumn{5}{|c|}{$A_{V}=5 \mathrm{mag}$} \\
\hline & $1 \%$ & $2 \%$ & $5 \%$ & $10 \%$ & $20 \%$ & $1 \%$ & $2 \%$ & $5 \%$ & $10 \%$ & $20 \%$ \\
\hline G0V $\left(M_{V}=+4.4\right)$ & 0.8 & 1.1 & 1.8 & 2.5 & 3.5 & 0.3 & 0.5 & 0.7 & 1.0 & 1.4 \\
\hline $\operatorname{K5III}\left(M_{V}=-0.1\right)$ & 1.3 & 2.6 & 4 & 7.5 & 11 & 1.0 & 1.5 & 2.4 & 3.5 & 5 \\
\hline Cepheid $\left(P=10^{\mathrm{d}}, M_{V}=-4.1\right)$ & 1.2 & 2.4 & 6 & 12 & 22 & 1.2 & 2.3 & 3.8 & 7 & 10 \\
\hline
\end{tabular}

maximum in 2012, which means that most of the radiation damage is likely to occur early in the mission.

Several methods are used to mitigate the effects of radiation damage, including shielding (which however is difficult to achieve for the expected proton energies over such a large focal plane), optimum choice of the CCD operating temperature $(\simeq 160 \mathrm{~K})$, and electronic charge injection (and/or a diffuse low-level background illumination of the CCDs) to fill the traps. However, detailed modelling of the effects as part of the data processing and calibration will also be necessary. On the positive side, extensive laboratory tests conducted by EADS Astrium on irradiated CCDs, operated under realistic conditions, have shown that the effects are highly deterministic and therefore possible to calibrate. The accuracy predictions take only part of the radiation damage into account, namely the charge loss. Remaining calibration errors have not been fully quantified yet but are targeted to contribute at modest levels to the end-of-mission performance.

Another critical calibration issue concerns the stability of the basic angle between the two viewing directions - nominally $106.5^{\circ}$. General fluctuations of the basic angle are passively controlled (to $<7 \mu$ as rms) by the thermo-mechanical design of the satellite. Moreover, on timescales longer than a few spin periods, the basic angle is accurately determined as part of the data processing. However, on timescales shorter than $\simeq 12$ hours the stability of the basic angle (or knowledge of its variations) is crucial for the global astrometry. In particular, as seen from Fig. 1 (right), a systematic variation in phase with the solar azimuth angle would be very detrimental to the parallax measurements, by producing a global parallax zero-point error that could not be detected from the residuals of the observations. An independent metrology device, the basic-angle monitor (BAM), is therefore in place to measure any short-term variations. It consists of two optical devices, each directing a pair of low-intensity laser beams towards its respective primary mirror. In the focal plane, the beams produce two sets of interference fringes that are detected by a separate CCD (Fig. 2). The devices are optically connected so that the angle between the two pairs of beams is insensitive to the relative orientation of the devices, and therefore to any deformation of the mechanical structure supporting them or the mirrors. The beams consequently form a stable reference against which any opto-mechanical changes can be detected as a relative displacement of the two sets of interference fringes. The BAM is capable of measuring basic-angle variations at the sub- $\mu$ as level every few minutes. By including these measurements in the data processing 
chain, the astrometric effects of short-term variations are eliminated. The BAM itself can be calibrated by means of other variations that are not in phase with the solar azimuth angle, and which produce recognizable signatures in the residuals of the observations.

\section{Conclusion}

Technically and scientifically, the Gaia project is well on track towards its scheduled launch around 2012. It is in many respects a difficult and challenging project, but the rewards in terms of the expected science return are overwhelming.

\section{Acknowledgements}

The present advanced state of the Gaia mission and its preparations is the result of a long and fruitful collaboration between many teams and individuals within ESA, industry and the scientific community. This review has freely used material from various sources and documents available within the Gaia community. Financial support by the national governments, research and space organizations to this community is gratefully acknowledged.

\section{References}

Aguilar, L. A., Brown, A. G. A., \& Velázquez, H. 2006, Rev. Mexicana AyA 26, 51

Bastian, U. 1995, in: M. A. C. Perryman \& F. van Leeuwen (eds.), Future Possibilities for Astrometry in Space, ESA SP-379, p. 99

de Bruijne, J. H. J. 2005, in: C. Turon, K. S. O'Flaherty, \& M. A. C. Perryman (eds.), The Three-Dimensional Universe with Gaia, ESA SP-576, p. 35

Cox, A. N. (ed.) 2000, Allen's astrophysical quantities, 4th ed. (Nex York: AIP Press; Springer)

Høg, E. 1993, in: I. I. Mueller \& B. Kołaczek (eds.), Developments in Astrometry and Their Impact on Astrophysics and Geodynamics, Proc. IAU Symposium No. 156 (Dordrecht: Kluwer), p. 37

Høg, E. 2008, this volume p.300

Mignard, F., et al. 2008, this volume, p.224

Mouret, S., Hestroffer, D., \& Mignard, F. 2007, A\&A 472, 1017

Straižys, V., Lazauskaitè, R., Brown, A. G. A., \& Zdanavičius, K. 2006, Baltic Astronomy 15, 449

Turon, C., O'Flaherty, K. S., \& Perryman, M. A. C. (eds.) 2005, The Three-Dimensional Universe with Gaia, ESA SP-576 